\title{
Preoperative Diagnosis of Suprasellar Hemangioblastoma with Four-Dimensional Computed Tomography Angiography: Case Report and Literature Review
}

\author{
Yi Tong ${ }^{1}$ Denis Sirhan ${ }^{2}$ Maria Cortes ${ }^{1,3}$ \\ ${ }^{1}$ Department of Radiology, McGill University Health Center, \\ Montreal, Quebec, Canada \\ ${ }^{2}$ Department of Neurosurgery, Montreal Neurological Hospital and \\ Institute, Montreal, Quebec, Canada \\ ${ }^{3}$ Department of Radiology, Montreal Neurological Hospital and \\ Institute, Montreal, Quebec, Canada \\ Indian J Radiol Imaging 2021;31:499-509.
}

\begin{abstract}
Address for correspondence Maria Cortes, MD, Department of Radiology, Montreal Neurological Hospital and Institute, 3801 University Street, Fifth Floor Radiology, Rm 540A, Montréal, QC H3A 2B4, Canada (e-mail: mariadelpilar.cortes@mcgill.ca).
\end{abstract}

\begin{abstract}
Purpose Our case report presents the first case of suprasellar hemangioblastoma diagnosed preoperatively with dynamic computed tomography angiography (four-dimensional [4D] CTA) in a patient without Von Hippel-Lindau (VHL) disease. We illustrate the imaging characteristics of these exceedingly rare tumors and discuss the role of 4D CTA in confirming this diagnosis and guiding surgical management. Finally, we present a literature review of imaging findings, differential diagnosis, management, and prognosis.

Case A 39-year-old woman known for diabetes mellitus type II and dyslipidemia presented with headache, bitemporal hemianopsia, and mild hyperprolactinemia. Initial diagnosis of suprasellar meningioma separate from pituitary gland was revised to

Keywords

- 4D CT angiography

- dynamic CT angiography

- hemangioblastoma

- sella turcica definitive diagnosis of suprasellar hemangioblastoma after 4D CTA.

Conclusion Suprasellar hemangioblastomas are extremely rare, often associated to VHL disease. They present as enhancing as suprasellar mass with prominent intra- and peritumoral vascular flow-voids on magnetic resonance imaging. 4D CTA confirms their vascular nature, demonstrates characteristic rapid shunting with feeding arteries, and enlarged draining veins, and is important in guiding surgical management.
\end{abstract}

\section{Key Messages}

Suprasellar hemangioblastomas present with enhancement and prominent vascular flow-voids on magnetic resonance imaging. Dynamic computed tomography angiography provides key information such as rapid intratumoral shunting, characteristic feeding arteries, and enlarged draining veins, and is important in surgical planning. Total resection is challenging, but when successful, no recurrence has been reported. Radiotherapy is an alternative treatment option.

\section{Introduction}

Suprasellar hemangioblastomas are exceedingly rare entities. In fact, hemangioblastomas most commonly arise in the posterior fossa, but are also found in the spinal cord and retina. ${ }^{1}$

(c) 2021. Indian Radiological Association.

This is an open access article published by Thieme under the terms of the Creative Commons Attribution-NonDerivative-NonCommercial-License, permitting copying and reproduction so long as the original work is given appropriate credit. Contents may not be used for commercial purposes, or adapted, remixed, transformed or built upon. (https://creativecommons.org/licenses/by-nc-nd/4.0/). Thieme Medical and Scientific Publishers Private Ltd. A-12, Second Floor, Sector -2, NOIDA -201301, India 
Hemangioblastomas are benign and highly vascularized neoplasms of unclear histological origin ${ }^{1}$ representing 1 to $2.5 \%$ of all primary intracranial neoplasms. Of note, $30 \%$ of hemangioblastomas are linked to Von Hippel-Lindau (VHL) disease. ${ }^{2}$ In these cases, the patient often has a known family history or personal history of hemangioblastomas or other stigmata. ${ }^{1}$

We present the first case of suprasellar hemangioblastoma diagnosed preoperatively based on four-dimensional computed tomography angiography (4D CTA) in an adult patient without VHL disease.

\section{Case History}

A 39-year-old woman, known for diabetes mellitus type II and dyslipidemia, presented with headache for the past few months. Family history was negative. Bitemporal hemianopsia was confirmed on visual field testing, without other focal neurological deficits. Endocrinological profile showed mild hyperprolactinemia (35 ug/L) (-Table 1). CT of the chest, abdomen, and pelvis with contrast were unremarkable.

Initial noncontrast CT scan of the head ( - Fig. 1) showed a solid, heterogeneous suprasellar mass with no cystic component, calcifications, cavernous sinus involvement, or hyperostosis. The mass was seen separate from normal pituitary gland and sella turcica was not enlarged. Based on imaging findings, a diagnosis of suprasellar mass like chiasmal glioma, suprasellar meningioma, or choroid glioma was offered. Magnetic resonance imaging (MRI) was done to further characterize the lesion.

MRI (-Figs. 2 and 3), including MR angiography (MRA) and venography ( - Fig. 4 ), was limited by motion artifact but revealed a $33 \times 25 \times 30 \mathrm{~mm}$ solid, heterogeneous mass. This lesion was isointense on T1-weighted images and hyperintense on T2-weighted images. Multiple associated hypointense rounded structures were consistent with peritumoral and intratumoral vascular flow-voids (-Figs. 2A, B and 3A, B). The lesion was avidly enhancing (-Figs. 2D and 3C, D). The mass was seen to compress hypothalamus, pituitary infundibulum, and antero-inferior recess of the third ventricle, with associated cerebral edema in the bilateral inferior

Table 1 Endocrinological profile on admission

\begin{tabular}{|l|l|l|}
\hline & $\begin{array}{l}\text { Patient's } \\
\text { value }\end{array}$ & $\begin{array}{l}\text { Institutional } \\
\text { normal range }\end{array}$ \\
\hline Prolactin (ug/L) & 35.2 & $3.3-26.7$ \\
\hline TSH (mlU/L) & 2.00 & $0.40-4.40$ \\
\hline Thyroxine (free) (pmol/L) & 8.20 & $8.00-18.00$ \\
\hline LH (IU/L) & 0.3 & N/A \\
\hline FSH (IU/L) & 0.6 & N/A \\
\hline GH (ug/L) & 0.10 & $0.03-4.00$ \\
\hline ACTH (pmol/L) & 3.32 & $1.60-13.90$ \\
\hline Cortisol AM (nmol/L) & 221 & $120-535$ \\
\hline Cortisol random (nmol/L) & 178 & $120-535$ \\
\hline
\end{tabular}

Abbreviations: ACTH, adrenocorticotropic hormone; FSH, follicle-stimulating hormone; $\mathrm{GH}$, growth hormone; $\mathrm{LH}$, luteinizing hormone; TSH, thyroid stimulating hormone.

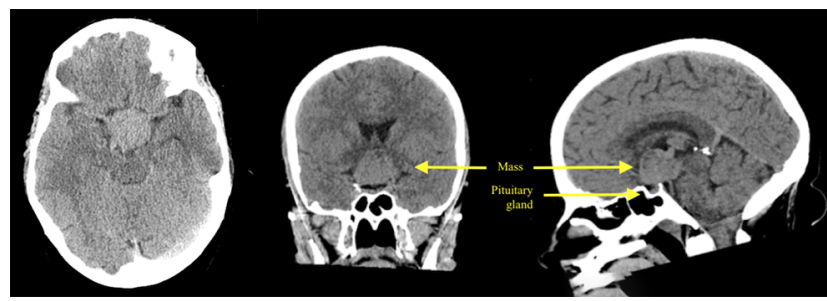

Fig. 1 Initial computed tomography (CT) head (noncontrast).

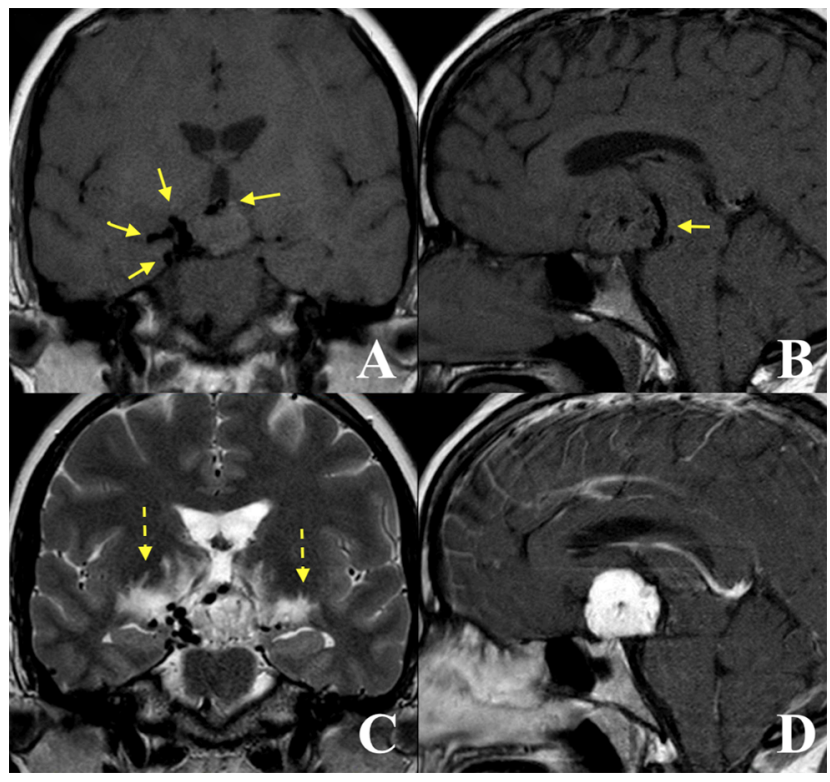

Fig. 2 Magnetic resonance imaging.

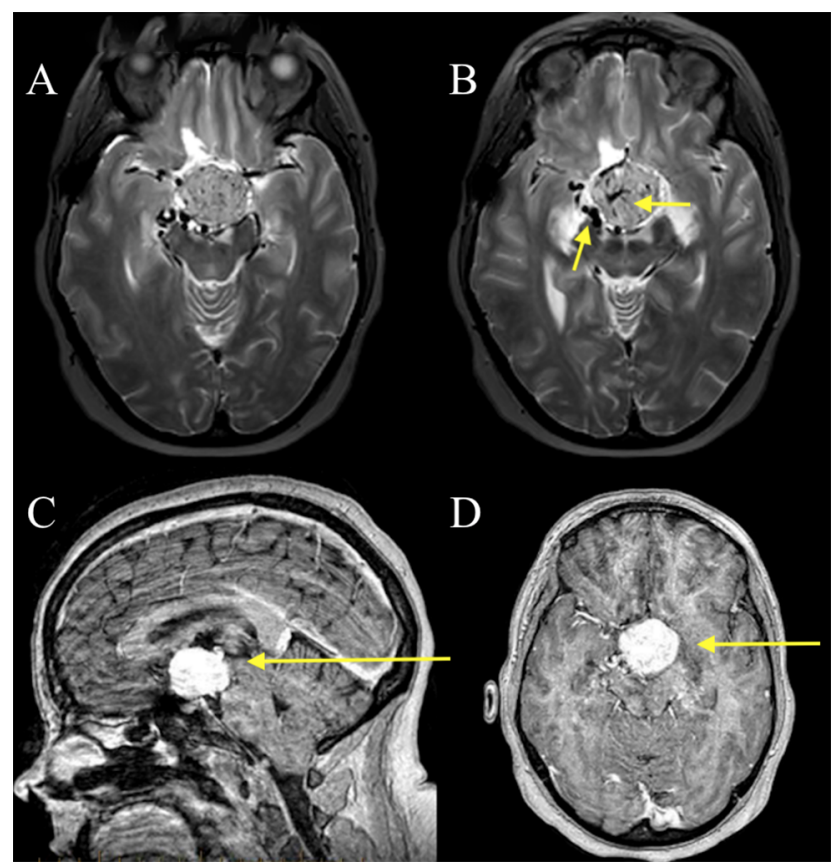

Fig. 3 Magnetic resonance imaging.

frontal regions and mesial temporal areas ( - Fig. 2C). The optic chiasm was clearly separate from the lesion. MRA demonstrated multiple intratumoral and peritumoral hyperintense 

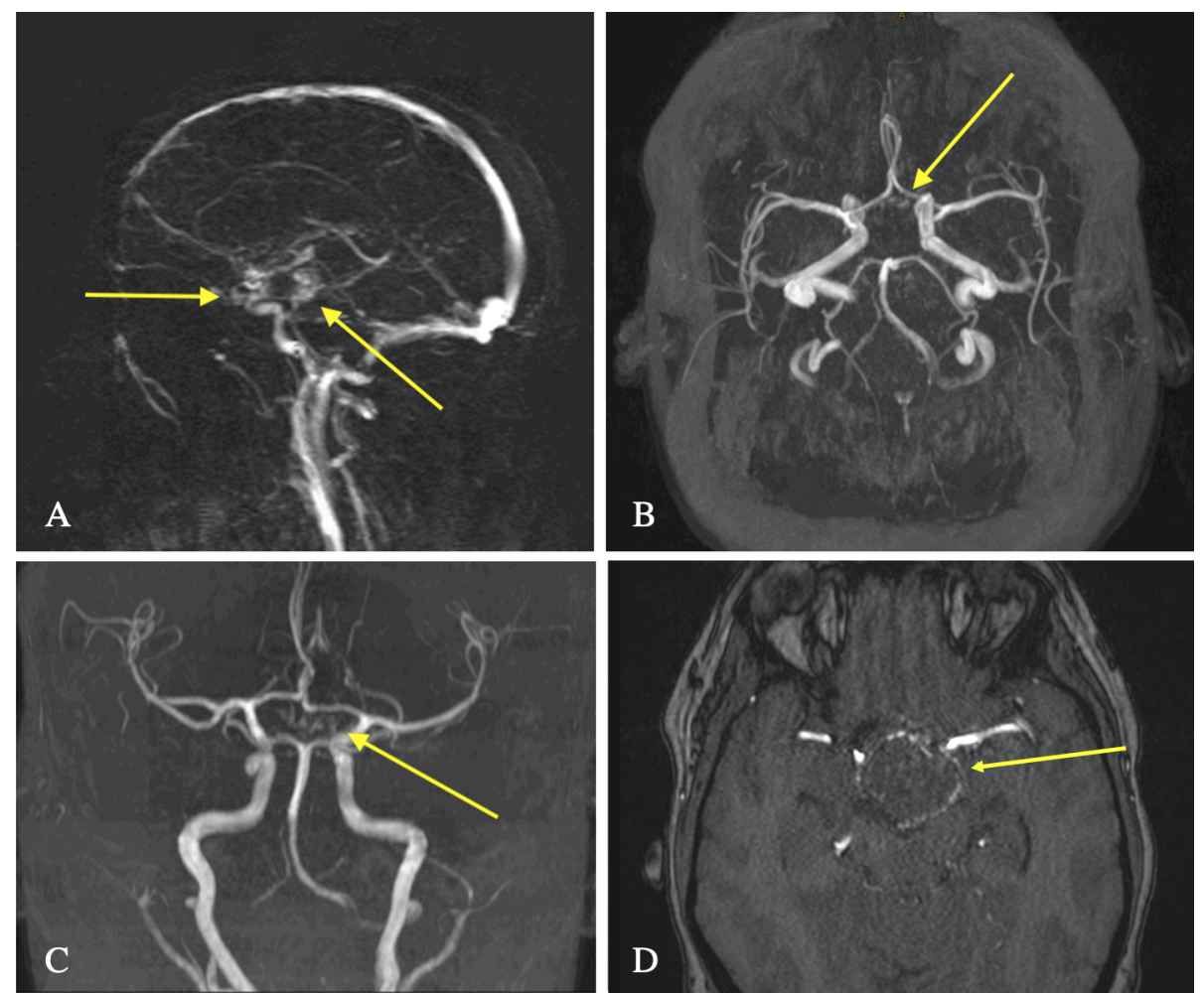

Fig. 4 Magnetic resonance angiography.

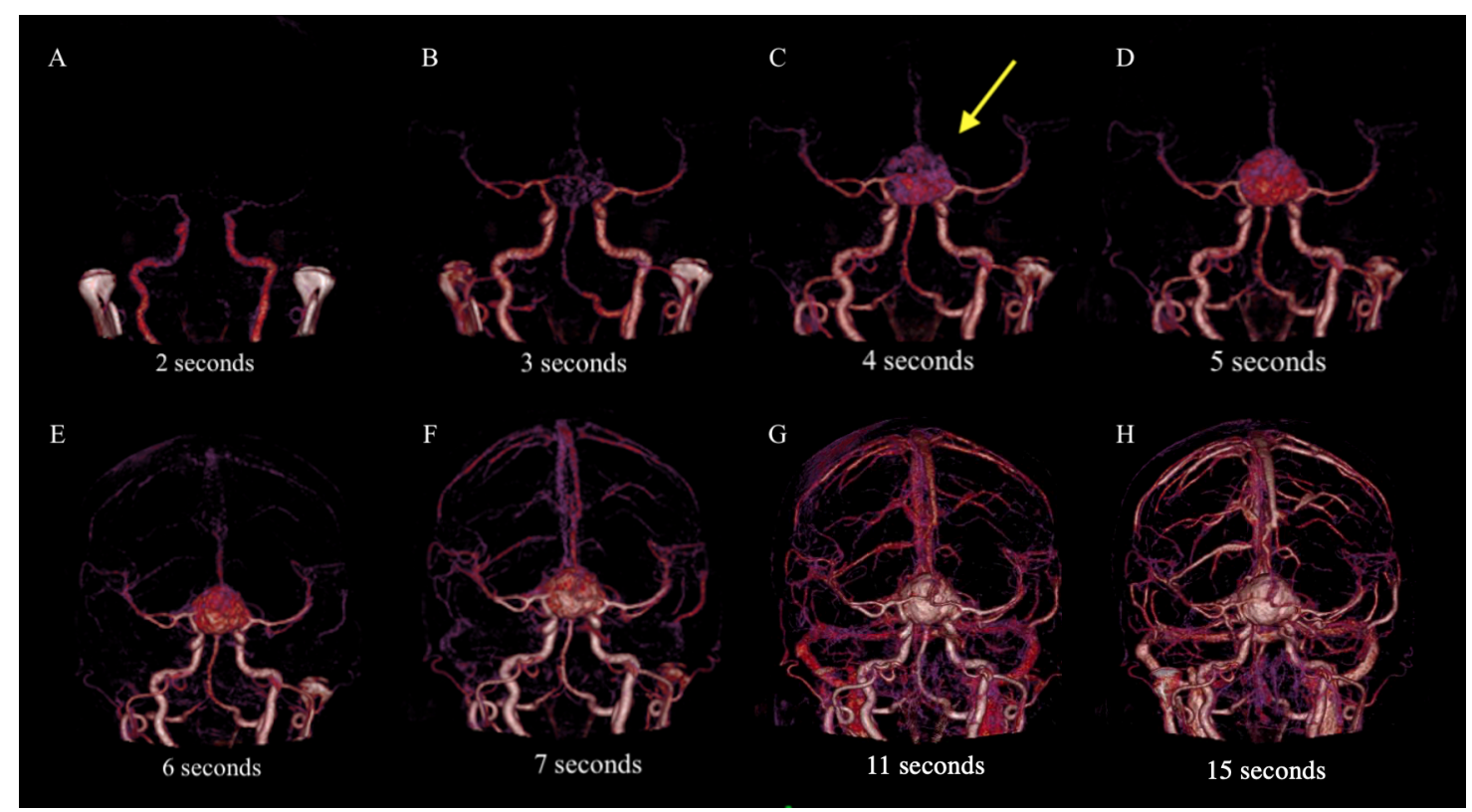

Fig. 5 CTA 4D images demonstrating arterial to venous phases with early contrast opacification of the suprasellar tumor.

curvilinear structures, consistent with high tumoral vascularity, without evidence of aneurysm or arteriovenous malformation (-Fig. 4).

In light of the findings on CT and MRI, a few differential diagnoses were considered, notably papillary subtype of craniopharyngioma, chiasmal-hypothalamic glioma, and meningioma arising from the dorsum sellae (see the "Discussion" section).
Due to atypical appearance on MRI and to better characterize the surrounding vasculature (notably the prominent draining veins) in prevision of a surgical intervention, the patient underwent dynamic CTA (4D CTA) (see Appendix A, "Materials and Methods" for technical details) which showed marked curvilinear enhancement around the mass, reflecting a combination of large feeding arteries and prominent venous drainage (-Fig. 5). The appearance suggested 

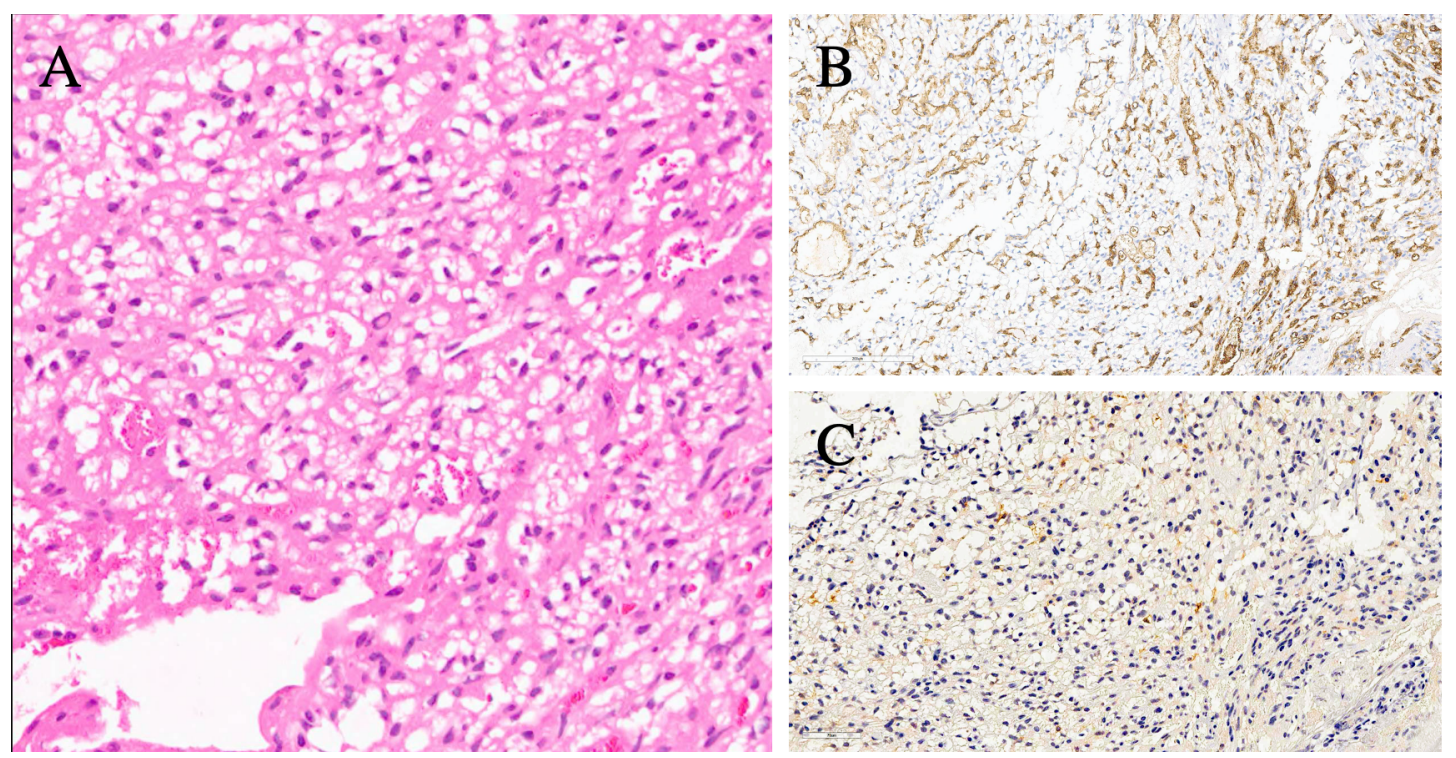

Fig. 6 Histopathology slides of the tumor. (A) H\&E stain showing a highly vascular tumor with thin walled capilaries. (B) Immunohistochemistry stain with CD34. (C) Inhibin stain positive confirmeing the diagnosis of hemangioblastoma.

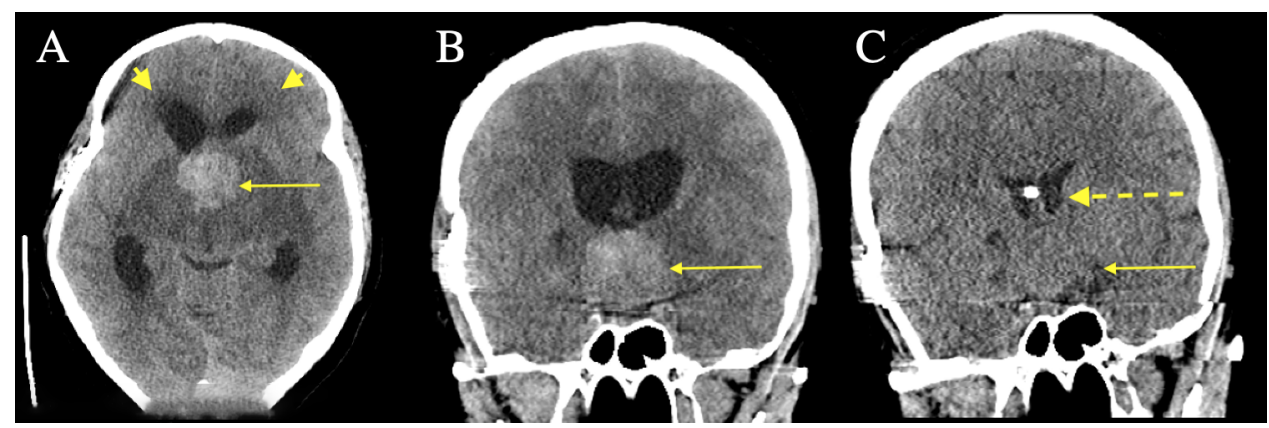

Fig. 7 CT Images of the brain, 9 months post operative, demonstrating intratumoral spontaneous bleeding and hydrocephalus.

very rapid intratumorally shunting, as one classically sees on angiographical studies of typical posterior fossa hemangioblastomas.

After 4D CTA, the report was revised to consider suprasellar hemangioblastoma as the most likely diagnosis and this was communicated to the neurosurgical team. The patient underwent right pterional and subfrontal craniotomy for tissue sampling and possible resection. Intraoperatively, the tumor was noted to be purple and vascular-appearing, adjacent to a highly prefixed chiasm. A biopsy of the tumor was taken. Given the intraoperative findings and preoperative radiological characteristics, all suggestive of high risk of hemorrhage, no resection was attempted. There were no intraoperative complications.

Immunohistochemistry confirmed the diagnosis of hemangioblastoma with focal positive staining for inhibin (-Fig. 6). The patient underwent stereotaxic radiosurgery 4 months after her operation. Unfortunately, her treatment was complicated by panhypopituitarism, leading to adrenal crisis.

Nine months after her craniotomy and biopsy, the patient presented with sudden onset of headache and vomiting. On nonenhanced CT ( - Fig. 7), findings were suggestive of acute rebleeding of the suprasellar hemangioblastoma with mass effect on the basal cisterns, as well as suspected intraventricular extension of the hemorrhage. There was associated findings of acute communicating hydrocephalus ( - Fig. 7), possibly secondary to the acute mass effect or due to intraventricular hemorrhagic components. The patient was treated with ventriculoperitoneal shunt placement. After further treatment with stereotaxic radiosurgery, the tumor minimally reduced in size ( - Fig. 7 ). At her last follow-up appointment, 12 months after her initial presentation, the patient was medically stable. Unfortunately, she was subsequently lost to follow-up.

\section{Discussion}

In the past 40 years, 33 cases of suprasellar hemangioblastomas have been reported in live patients (-Table 2): 19 of these cases have been patients with VHL and 12 cases of patients without VHL, with two cases of unclear VHL status. Overall, $30 \%$ of hemangioblastomas are associated with VHL, but a sellar or suprasellar location seems to have a stronger association to the disease.,3 Our patient's symptoms were congruent with the most commonly reported symptoms ("visual disturbances" and "headache") (-Table 2). 
Table 2 Reported cases of suprasellar hemangioblastoma in the literature

\begin{tabular}{|c|c|c|c|c|c|c|c|c|}
\hline & Patient & Presentation & VHL & Imaging & Preop Dx & Treatment & Follow-up & Year, Ref \\
\hline 1 & $19 M$ & $\begin{array}{l}\text { Vertigo, vomiting, } \\
\text { ataxia, nystagmus }\end{array}$ & + & $\begin{array}{l}\text { CT: enhancing solid } \\
\text { nodule on the right } \\
\text { anterior edge of the } \\
\text { suprasellar cistern } \\
\text { Angio: supraclinoid } \\
\text { nodule }\end{array}$ & $N / A$ & \begin{tabular}{|l} 
Craniotomy \\
(total excision) \\
Sacrifice of optic \\
nerve
\end{tabular} & $\mathrm{N} / \mathrm{A}$ & $1981^{4}$ \\
\hline 2 & $28 \mathrm{~F}$ & $\begin{array}{l}\text { Headache, } \\
\text { amenorrhea, } \\
\text { galactorrhea }\end{array}$ & - & $\begin{array}{l}\text { CT: enhancing solid } \\
\text { Angio: highly vascu- } \\
\text { larized with persistent } \\
\text { blush }\end{array}$ & Meningioma & $\begin{array}{l}\text { Right frontal } \\
\text { craniotomy (total } \\
\text { excision) }\end{array}$ & $\begin{array}{l}\text { Alive at } 2 \text { mo } \\
\text { (panhypopit) }\end{array}$ & $1984^{5}$ \\
\hline 3 & $60 \mathrm{~F}$ & $\begin{array}{l}\text { Partial } \\
\text { hemianopsia }\end{array}$ & + & $\begin{array}{l}\text { CT: } 24 \text { mm suprasellar } \\
\text { solid mass }\end{array}$ & $\mathrm{HBL}$ & Radiosurgery & $\begin{array}{l}\text { Alive at } 28 \text { mo, } \\
\text { SIADH, tumor } \\
\text { reduced by } \\
54 \%\end{array}$ & $1996^{6}$ \\
\hline 4 & $20 \mathrm{~F}$ & $\begin{array}{l}\text { Secondary amen- } \\
\text { orrhea, polydipsia }\end{array}$ & + & $\begin{array}{l}\text { MRI: 6-mm con- } \\
\text { trast-enhancing mass } \\
\text { of the tuber cinereum, } \\
\text { immediately posterior } \\
\text { to optic chiasm and } \\
\text { two enhancing masses } \\
\text { in right cerebellar } \\
\text { hemisphere }\end{array}$ & $\mathrm{HBL}$ & $\begin{array}{l}\text { Modified } \\
\text { transsphenoidal } \\
\text { approach }\end{array}$ & $\begin{array}{l}\text { No residual } \\
\text { tumor at } 53 \\
\text { mo (panhypo- } \\
\text { pit and DI) }\end{array}$ & $2000^{7}$ \\
\hline 5 & $15 \mathrm{~F}$ & Asymptomatic & + & $\begin{array}{l}\text { MRI: } 7 \times 14 \mathrm{~mm} \\
\text { enhancing lesion } \\
\text { compressing left } \\
\text { optic nerve and } 1 \mathrm{~cm} \\
\text { enhancing in vermis }\end{array}$ & $\mathrm{HBL}$ & $\begin{array}{l}\text { Modified } \\
\text { transsphenoidal } \\
\text { approach }\end{array}$ & $\begin{array}{l}\text { No residual } \\
\text { tumor at } 12 \\
\text { mo (CSF leak } \\
\text { POD \#6) }\end{array}$ & $2000^{7}$ \\
\hline 6 & $33 \mathrm{~F}$ & $\begin{array}{l}\text { Menses irreg- } \\
\text { ularity. Mildly } \\
\text { abnormal GnRH } \\
\text { stimulation test }\end{array}$ & + & $\begin{array}{l}\text { MRI: } 8 \text { mm round, } \\
\text { homogeneous } \\
\text { enhancement, T1 } \\
\text { isointense, T2 cystic } \\
\text { Angio: tumor blush } \\
\text { fed by superior hypo- } \\
\text { physeal artery }\end{array}$ & $\mathrm{HBL}$ & $\begin{array}{l}\text { Right frontotem- } \\
\text { poral craniotomy }\end{array}$ & $\begin{array}{l}\text { No residual } \\
\text { tumor at } 6 \text { mo }\end{array}$ & $2001^{8}$ \\
\hline 7 & $62 \mathrm{M}$ & Visual disturbances & - & $\begin{array}{l}\text { Angio: “remarkable } \\
\text { tumor staining” orig- } \\
\text { inating from the right } \\
\text { and left ICA and the } \\
\text { left ECA } \\
\text { MRI: homogeneous } \\
\text { enhancement, T2 } \\
\text { hyperintense }\end{array}$ & Meningioma & Craniotomy & $\begin{array}{l}\text { Paraparesis } \\
\text { POD \#7 }\end{array}$ & $2001^{9}$ \\
\hline 8 & $40 \mathrm{~F}$ & $\begin{array}{l}\text { Oligomenorrhea, } \\
\text { memory lapses }\end{array}$ & + & $\begin{array}{l}\text { MR: } 3.2 \mathrm{~cm} \text { solid, } \\
\text { homogeneous } \\
\text { enhancement } \\
\text { T1 isointense, T2 het- } \\
\text { erogeneous (hyper/ } \\
\text { iso), intratumoral } \\
\text { flow-void } \\
\text { Angio: hypervascular } \\
\text { mass, fed by small } \\
\text { perforators from distal } \\
\text { ICA and thalamoperfo- } \\
\text { rating arteries }\end{array}$ & $\begin{array}{l}\mathrm{HBL} \\
\text { (previous cerebellar) }\end{array}$ & $\begin{array}{l}\text { Subtotal excision } \\
\text { + radiosurgery }\end{array}$ & $\mathrm{N} / \mathrm{A}$ & $2003^{10}$ \\
\hline 9 & $54 \mathrm{M}$ & $\begin{array}{l}\text { Temporal hemi- } \\
\text { anopsia left + } \\
\text { complete visual } \\
\text { loss right }\end{array}$ & - & $\begin{array}{l}\text { MRI: Homogeneous } \\
\text { marked enhancement } \\
\text { T1 Isointense, T2 } \\
\text { hyperintense }\end{array}$ & Meningioma & $\begin{array}{l}\text { Right pterional } \\
\text { craniotomy }\end{array}$ & $\begin{array}{l}\text { No residual } \\
\text { tumor at } 5 \text { y }\end{array}$ & $2004^{11}$ \\
\hline 10 & $38 \mathrm{M}$ & $\begin{array}{l}\text { Complete visual } \\
\text { loss (left eye), } \\
\text { severe headaches }\end{array}$ & + & $\begin{array}{l}\text { MRI: Homogeneous } \\
\text { enhancement } \\
\text { T1 Isointense, T2 } \\
\text { hyperintense }\end{array}$ & $\begin{array}{l}\text { HBL (previous } \\
\text { cerebellar) }\end{array}$ & $\begin{array}{l}\text { Right pterional } \\
\text { craniotomy } \\
\text { (subtotal) }\end{array}$ & $\mathrm{N} / \mathrm{A}$ & $2004^{11}$ \\
\hline
\end{tabular}


Table 2 (Continued)

\begin{tabular}{|c|c|c|c|c|c|c|c|c|}
\hline & Patient & Presentation & VHL & Imaging & Preop Dx & Treatment & Follow-up & Year, Ref \\
\hline 11 & $51 \mathrm{~F}$ & $\begin{array}{l}\text { Progressive right } \\
\text { inferior temporal } \\
\text { quadranopsia }\end{array}$ & + & $\begin{array}{l}\text { MRI: } 20 \text { mm diameter, } \\
\text { T1 isointense homog- } \\
\text { enous, T2 hyperin- } \\
\text { tense, strong contrast } \\
\text { enhancement }\end{array}$ & $\begin{array}{l}\mathrm{HBL} \text { (previous retinal } \\
\text { and spinal } \mathrm{HBL} \text { ) }\end{array}$ & $\begin{array}{l}\text { Left frontopteri- } \\
\text { onal craniotomy }\end{array}$ & $\begin{array}{l}\text { 8-year } \\
\text { follow-up: } \\
\text { panhypopit, } \\
\text { no pituitary } \\
\text { recurrence, } 5 \\
\text { new cerebellar } \\
\text { HBLs (RadioTx) }\end{array}$ & $2007^{12}$ \\
\hline 12 & $59 F$ & $\begin{array}{l}\text { Fatigue, decreased } \\
\text { vision, somno- } \\
\text { lence, impaired } \\
\text { memory }\end{array}$ & $?$ & $\begin{array}{l}\text { Angio: blush from } \\
\text { both superior hypo- } \\
\text { physeal arteries } \\
\text { MRI: } 3.5-c m \text { enhanc- } \\
\text { ing lesion, small cystic } \\
\text { structures, T1 isoin- } \\
\text { tense, T2 hyperintense }\end{array}$ & $N / A$ & $\begin{array}{l}\text { Bifrontal inter- } \\
\text { hemispheric } \\
\text { craniotomy }\end{array}$ & $\begin{array}{l}\text { No residual } \\
\text { tumor at } 3 \text { y } \\
\text { (panhypopit) }\end{array}$ & $2008^{13}$ \\
\hline $\begin{array}{l}13- \\
20\end{array}$ & \begin{tabular}{|l|}
$38 \pm$ \\
$13 \mathrm{y}$ \\
4 \\
female4 \\
male
\end{tabular} & $\begin{array}{l}\text { Asymptomatic, } \\
\text { normal endocrine } \\
\text { profile, normal } \\
\text { visual testing }\end{array}$ & + & $\begin{array}{l}\text { MRI: mean tumor vol- } \\
\text { ume } 0.5 \pm 0.9 \mathrm{~cm}^{3}\end{array}$ & N/A & Observation & $\begin{array}{l}\text { Mean fol- } \\
\text { low-up } 41.4 \pm \\
14.4 \text { mo, no } \\
\text { deficits }\end{array}$ & $2009^{14}$ \\
\hline 21 & $30 \mathrm{M}$ & $\begin{array}{l}\text { Progressive vision } \\
\text { loss }\end{array}$ & + & No details provided & $N / A$ & $\begin{array}{l}\text { Craniotomy but } \\
\text { no attempt at } \\
\text { resection }\end{array}$ & $\begin{array}{l}\text { Death at } 120 \\
\text { mo (cause } \\
\text { directly linked } \\
\text { to } \mathrm{HBL} \text { ) }\end{array}$ & $2010^{15}$ \\
\hline 22 & $28 \mathrm{~F}$ & $\begin{array}{l}\text { 27-mo history of } \\
\text { galactorrhea and } \\
\text { alopecia }\end{array}$ & + & $\begin{array}{l}\text { MRI: } 4 \times 2 \times 2 \mathrm{~mm} \\
\text { pituitary infundibulum } \\
\text { mass with rapid con- } \\
\text { trast enhancement }\end{array}$ & $\begin{array}{l}\text { HBL (previous spinal } \\
\text { and cerebellar HBLs } \\
\text { resected) }\end{array}$ & Bromocriptine & $\begin{array}{l}\text { Galactorrhea } \\
\text { resolved } \\
\text { after } 2 \text { wk of } \\
\text { bromocriptine }\end{array}$ & $2010^{16}$ \\
\hline 23 & $80 \mathrm{~F}$ & $\begin{array}{l}\text { Episode of severe } \\
\text { headache with } \\
\text { nausea, vomit- } \\
\text { ing, and blurred } \\
\text { vision (pituitary } \\
\text { apoplexy). Known } \\
\text { 12-year history of } \\
\text { stable sellar mass }\end{array}$ & - & $\begin{array}{l}\text { MRI: acute hemor- } \\
\text { rhage of sellar mass }\end{array}$ & Pituitary adenoma & $\begin{array}{l}\text { Transnasal, trans- } \\
\text { sphenoidal total } \\
\text { resection }\end{array}$ & $\begin{array}{l}\text { Panhypopit } \\
\text { with tran- } \\
\text { sient DI. No } \\
\text { recurrence at } \\
16 \text { mo }\end{array}$ & $2011^{17}$ \\
\hline 24 & $12 \mathrm{~F}$ & $\begin{array}{l}\text { Headache and } \\
\text { bitemporal } \\
\text { hemianopsia, } \\
\text { hypopituitarism }\end{array}$ & - & $\begin{array}{l}\text { MRI: homogeneous } \\
\text { contrast enhancement } \\
\text { and optic chiasm } \\
\text { compression, intratu- } \\
\text { moral and peritumoral } \\
\text { engorged vessels }\end{array}$ & Pituitary adenoma & $\begin{array}{l}2 \text { attempts } \\
\text { (transsphenoidal } \\
\text { and transcranial), } \\
\text { stopped for } \\
\text { bleeding }\end{array}$ & $\begin{array}{l}\text { Clinically } \\
\text { improved at } \\
3 \text { mo }\end{array}$ & $2012^{18}$ \\
\hline 25 & $64 \mathrm{~F}$ & $\begin{array}{l}\text { Headache, bitem- } \\
\text { poral hemianopsia } \\
\text { Normal endo }\end{array}$ & - & $\begin{array}{l}\text { CT: solid suprasellar } \\
\text { mass of } 2.5 \mathrm{~cm} \\
\text { diameter } \\
\text { MRI: T1 isointense, T2 } \\
\text { hyperintense, curvilin- } \\
\text { ear areas of flow-void, } \\
\text { strong enhancement, } \\
\text { with cystic component } \\
\text { CT angio: supplied by } \\
\text { multiple small perfo- } \\
\text { rating arteries from } \\
\text { ACA and PCom }\end{array}$ & Craniopharyngioma & $\begin{array}{l}\text { Endoscopic } \\
\text { endonasal subto- } \\
\text { tal resection }\end{array}$ & $\begin{array}{l}1 \text { mo postop: } \\
\text { subtotal } \\
\text { resection. } \\
\text { CSF leak and } \\
\text { hydrocephalus } \\
\text { (VP shunt) }\end{array}$ & $2013^{19}$ \\
\hline 26 & $60 \mathrm{~F}$ & $\begin{array}{l}\text { Headache, } \\
\text { dizziness }\end{array}$ & - & $\begin{array}{l}\text { MRI: T1 isointense, } \\
\text { T2 heterogeneous, } \\
\text { multiple signal voids } \\
\text { inside mass, marked } \\
\text { homogeneous con- } \\
\text { trast enhancement }\end{array}$ & N/A & $\begin{array}{l}\text { Right frontotem- } \\
\text { poral craniotomy }\end{array}$ & $\begin{array}{l}\text { Transient DI, } \\
\text { no neuro or } \\
\text { endo deficits } \\
\text { at } 1 \mathrm{y}\end{array}$ & $2015^{20}$ \\
\hline
\end{tabular}


Table 2 (Continued)

\begin{tabular}{|c|c|c|c|c|c|c|c|c|}
\hline & Patient & Presentation & VHL & Imaging & Preop Dx & Treatment & Follow-up & Year, Ref \\
\hline 27 & $51 \mathrm{~F}$ & $\begin{array}{l}\text { Headache, left } \\
\text { visual field defect }\end{array}$ & - & $\begin{array}{l}\text { MRI: solid mass, T1 } \\
\text { isointense, T2 hyper- } \\
\text { intense with homo- } \\
\text { geneous contrast } \\
\text { enhancement }\end{array}$ & N/A & $\begin{array}{l}\text { Left pterional } \\
\text { craniotomy }\end{array}$ & $\begin{array}{l}\text { No residual at } \\
1 \text { y (transient } \\
\text { DI) }\end{array}$ & $2015^{2}$ \\
\hline 28 & $35 \mathrm{~F}$ & $\begin{array}{l}\text { Neurofibromatosis } \\
1 \text {, headache, near } \\
\text { complete visual } \\
\text { loss right eye }\end{array}$ & - & $\begin{array}{l}\text { MRI: } 8 \text { mm lesion with } \\
\text { small cystic compo- } \\
\text { nents, T1 isointense, } \\
\text { T2 hyperintense, } \\
\text { marked contrast } \\
\text { enhancement }\end{array}$ & & \begin{tabular}{|l|} 
Endoscopic \\
expanded \\
transsphenoidal \\
resection with \\
sphenoidectomy
\end{tabular} & $\begin{array}{l}\text { Transient DI } \\
\text { (lasting } 4 \mathrm{mo} \text { ), } \\
\text { stable } 11 \mathrm{mo} \\
\text { postop }\end{array}$ & $2016^{21}$ \\
\hline 29 & $67 \mathrm{~F}$ & $\begin{array}{l}\text { Retro-orbital pain, } \\
\text { bilateral upper } \\
\text { temporal quadran- } \\
\text { tanopsia, mild DI }\end{array}$ & - & $\begin{array}{l}\text { MRI: Significant } \\
\text { enhancement, slight } \\
\text { upward displacement } \\
\text { of optic chiasm }\end{array}$ & N/A & $\begin{array}{l}\text { Transsphenoidal } \\
\text { resection }\end{array}$ & $\mathrm{DI}$ & $2017^{22}$ \\
\hline 30 & $64 \mathrm{~F}$ & $\begin{array}{l}\text { Left inferior quad- } \\
\text { rantanopsia, (right } \\
\text { eye enucleated) }\end{array}$ & + & $\begin{array}{l}\text { MRI: T1 avid enhance- } \\
\text { ment, no other details } \\
\text { reported }\end{array}$ & N/A & $\begin{array}{l}\text { Octreotide } \\
\text { intramuscular } \\
\text { (unresectable) }\end{array}$ & $\begin{array}{l}\sim 25 \% \text { decrease } \\
\text { in tumor } \\
\text { volume after } \\
9 \text { mo }\end{array}$ & $2017^{23}$ \\
\hline 31 & $38 \mathrm{~F}$ & $\begin{array}{l}9 \text { mo history of } \\
\text { amenorrhea with } \\
\text { low LH and FSH, } \\
\text { headaches }\end{array}$ & $?$ & $\begin{array}{l}\text { MRI: } 13 \times 13 \times 13.2 \\
\text { mm mass in upper } \\
\text { half of infundibulum, } \\
\text { T1 isointense, T2 } \\
\text { isointense, avid con- } \\
\text { trast enhancement, } \\
\text { unremarkable on DWI } \\
\text { and ADC map }\end{array}$ & Pituicytoma & $\begin{array}{l}\text { Endoscopic trans- } \\
\text { sphenoidal total } \\
\text { resection }\end{array}$ & $N / A$ & $2017^{24}$ \\
\hline 32 & $60 \mathrm{~F}$ & $\begin{array}{l}\text { Headache, abdu- } \\
\text { cens palsy, low } \\
\text { ATCH }\end{array}$ & - & $\begin{array}{l}\text { MRI: } 14 \times 12 \mathrm{~mm} \text {, } \\
\text { rounded mass } \\
\text { attached to the } \\
\text { pituitary stalk, avidly } \\
\text { enhancing, multiple } \\
\text { flow-voids, fed by } \\
\text { short perforators from } \\
\text { the left ICA and pos- } \\
\text { terior communicating } \\
\text { artery }\end{array}$ & $\mathrm{HBL}$ & $\begin{array}{l}\text { Right OZ } \\
\text { craniotomy }\end{array}$ & $\begin{array}{l}\text { Stable at } 36 \\
\text { mo, improved } \\
\text { vision and } \\
\text { endocrine } \\
\text { function }\end{array}$ & $2018^{25}$ \\
\hline 33 & $28 \mathrm{~F}$ & $\begin{array}{l}\text { Bitemporal } \\
\text { hemianopsia }\end{array}$ & - & $\begin{array}{l}\text { CT: no calcification } \\
\text { MRI: } 10 \times 7 \times 12 \mathrm{~mm} \\
\text { solid tumor with } \\
\text { accompanying } 10 \mathrm{~mm} \\
\text { cystic component, } \\
\text { T1 isointense T2 } \\
\text { hyperintense, avidly } \\
\text { enhancing, edema-like } \\
\text { change along the optic } \\
\text { tract }\end{array}$ & Craniopharyngioma & $\begin{array}{l}\text { Biopsy attempt } \\
\text { (excess bleed > } 1 \\
\text { L) then complete } \\
\text { excision }\end{array}$ & $\begin{array}{l}\text { No tumor } \\
\text { recurrence at } \\
6 \text { mo }\end{array}$ & $2018^{26}$ \\
\hline
\end{tabular}

Abbreviations: ACA, anterior cerebral artery; ACTH, adrenocorticotropic hormone; ADC, apparent diffusion coefficient; CSF, cerebrospinal fluid; CT, computed tomography; DI, diabetes insipidus; DWI, diffusion-weighted imaging; Dx, diagnosis; ECA, external carotid artery; FSH, follicle-stimulating hormone; $\mathrm{GnRH}$, gonadotropin releasing hormone; HBL, hemangioblastoma; ICA, internal carotid artery; LH, luteinizing hormone; MRI, magnetic resonance imaging; OZ, orbitozygomatic; panhypopit, panhypopituitarism; PCom, posterior communicating artery; POD, postop day; SIADH, syndrome of inappropriate antidiuretic hormone; VHL, Von Hippel-Lindau; VP, ventriculoperitoneal.

This case is the first reported preoperatively diagnosed suprasellar hemangioblastoma confirmed on 4D CTA in a patient with no evidence of VHL disease. In such sporadic cases, previous reports describe preoperative diagnoses like craniopharyngioma, pituitary adenoma, pituicytoma, and meningioma. ${ }^{5,9,11,13,18,19,26}$ Our case's unusual imaging features prompt consideration of less frequent suprasellar tumors like papillary craniopharyngioma, angiomatous meningioma, hemangiopericytoma, as well as chordoid glioma.
The rare papillary subtype represents about one-third of adult craniopharyngiomas. These lesions are predominantly solid or mixed solid-cystic and have a predilection to involve the third ventricle. Notably, papillary craniopharyngiomas do not contain calcifications. On MRI, the usual appearance is hypointense on T1-weighted images, hyperintense on T2-weighted images, with cyst wall enhancement postgadolinium. ${ }^{27,28}$ In our case, papillary subtype of craniopharyngioma was considered in the differential but the 
significant presence of intratumoral and peritumoral vascular flow-voids was deemed atypical.

As for angiomatous meningioma, a very rare histological subtype of meningiomas, they have a male predominance. These dural-based lesions can arise from the skull base, with high attenuation on CT. The angiomatous histological type appears hypointense on T1-weighted images, hyperintense on T2-weighted images, and hypointense on diffusion-weighted imaging. Due to prominent hypervascularity, it enhances avidly with internal vascular flow-voids and surrounding brain edema. Other features may be present, such as a dural tail, involvement or encasement of the cavernous internal carotid artery, and bone erosion. ${ }^{29,30}$ In our case, the diagnosis of a highly vascular meningioma was entertained, given the location and MRI appearance. However, there was notable absence of associated findings such as a dural tail, internal carotid artery involvement, or bone erosion.

Another uncommon lesion to consider would be hemangiopericytoma, a tumor that tends to be large and lobulated in appearance, with intense but heterogeneous enhancement. It rarely shows calcifications but is frequently associated with peritumoral edema. ${ }^{31-33} \mathrm{Up}$ to $20 \%$ of cases have malignant behavior, with possible metastasis outside of the central nervous system (bone, liver, lungs). ${ }^{31,32}$ On MRI, hemangiopericytomas are usually isointense with heterogeneous contrast enhancement ${ }^{31,32}$ and prominent internal signal voids. ${ }^{32} \mathrm{~A}$ dural tail is seen in approximately $50 \%$ of cases. ${ }^{31}$ More than half of cases have bone erosion. ${ }^{32}$ The lesion of our presented case did not have a dural tail and lacked aggressive features such as bone erosion. Our patient had no evidence of metastatic lesions on MRI of the head and CT of the chest, abdomen, and pelvis.

Finally, a differential diagnosis for lesions near the third ventricle includes chordoid gliomas, which predominantly occur in adult women. On MRI, this tumor shows a well-defined ovoid mass in the anterior third ventricle or sellar region. It is isointense on T1-weighted images with uniform contrast enhancement and bilateral vasogenic edema. On CT, it is hyperdense to gray matter. ${ }^{34}$ However, a main feature of the tumor in our case is the presence of prominent vascular flow-voids, which is not typical for chordoid gliomas.

Thus, the preoperative diagnosis of suprasellar hemangioblastoma is challenging due to its rarity and the many other entities to be considered in the differential, as detailed above. Our case presents typical MRI features of hemangioblastomas: isointense on T1-weighted images, hyperintense on T2-weighted images, with marked contrast enhancement (-Table 2). These lesions can also have a cystic appearance, but surrounding edema, as in our case, is more atypical. Most importantly, hemangioblastomas have prominent vascular flow-voids on MRI.

Although MRI and MRA provide high-quality visualization of the intracranial arteries, the appearance of vascular lesions is influenced by size, flow direction, pulsatility, flow velocity, and degree of thrombosis. ${ }^{35-38} 4 \mathrm{D}$ CTA is known to have excellent spatial and temporal resolution and is uniquely helpful in evaluating structures of the skull base. ${ }^{35,36}$ In addition, 4D
CTA is reported to have a better sensitivity than MRA with better visualization of surrounding vessel anatomy and is less prone to flow-related or motion artifacts. ${ }^{36}$ Moreover, in our case, 4D CTA was essential in evaluating venous drainage, and in general, is very helpful in planning interventions on complex skull base tumors. ${ }^{36} 4 \mathrm{D}$ CTA is a less invasive modality than traditional angiography (digital subtraction angiography [DSA]), with near-equal sensitivity and specificity in the evaluation of aneurysms, ${ }^{37}$ but DSA remains the gold standard when evaluating vascular neoplasms of the head and neck.

For a hemangioblastoma, 4D CTA will clearly demonstrate characteristic features of very rapid shunting, large feeding arteries with dilated draining veins, and a deep tumor blush. The early venous shunting confirmed rapid flow within the tumor, and also helped for the surgical planning. In terms of differential diagnosis, the appearance of meningioma on $4 \mathrm{D}$ CTA is different, typically featuring a persistent tumor blush with delayed washout, in contrast with the early venous drainage of hemangioblastoma. ${ }^{39}$ Moreover, a meningioma classically features a central vascular pedicle from which smaller vessels radiate ("spoke wheel" appearance) and often features vascular supply from prominent meningeal or pial vessels. ${ }^{39}$ The high-resolution dynamic characteristics of $4 \mathrm{D}$ CTA provide added diagnostic benefit compared with MRI and MRA. In our case, 4D CTA was deemed to have provided adequate quality of dynamic diagnostic information and DSA was not performed. Of note, $4 \mathrm{D}$ contrast-enhanced MRA is another imaging modality that can provide reliable hemodynamic diagnostic information in head and neck tumors. Even though 4D contrast-enhanced MRA is useful in characterizing tumor stain, this modality is not a replacement to DSA due to poorer temporal resolution (more specifically, poorer identification of feeding arteries). ${ }^{40}$

Preoperative diagnosis of hemangioblastoma is important for surgical management. Resection of suprasellar hemangioblastomas is often limited by surrounding structures $^{4}$ and risk of hemorrhage. ${ }^{18,26}$ In all cases of successful

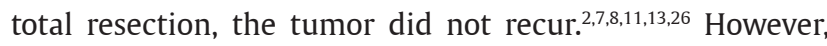
important surgical complications include cerebrospinal fluid leak, ${ }^{7,19}$ hydrocephalus, ${ }^{19}$ and paraparesis. ${ }^{9}$ Postoperative endocrine dysfunction can include panhypopituitarism, ${ }^{5,8,35}$ diabetes insipidus, ${ }^{2,6}$ and syndrome of inappropriate antidiuretic hormone. ${ }^{6}$ The option of preoperative embolization is mitigated by risks of infarct. ${ }^{41}$ Targeted radiotherapy has shown good results in tumor volume reduction. ${ }^{74}$ Intramuscular octreotide in a small group of VHL patients with hemangioblastomas expressing somatostatin receptors has also been linked to significant tumor volume reduction; however, this remains experimental. ${ }^{23}$

\section{Conclusion}

Suprasellar hemangioblastoma is an extremely rare diagnosis and presents as a mass with prominent vascular flow-voids on MRI. 4D CTA can confirm the vascular nature of the lesion and characteristic features of very rapid shunting, large feeding arteries, dilated draining veins, and deep tumor blush. 
Dynamic imaging also helps in guiding the surgical approach and can influence intraoperative decisions. A hemangioblastoma located in the sellar-suprasellar region is often associated to VHL disease. Surgical resection is complex due to tumor location. Benefits must be weighed against the high risk of hemorrhage and other postoperative complications. However, there are no reported cases of recurrence after total resection. Treatment with radiotherapy is another option.

\section{Presentation at a Meeting}

American Society of Neuroradiology 2019 Annual Meeting, Boston, MA, USA, May 20th, 2019.

\section{Source(s) of Support}

None to declare.

\section{Conflict of Interest}

None declared.

\section{Acknowledgments}

None.

\section{References}

1 Metelo A, Iliopoulos O, Hemangioblastomas of the Central Nervous System. In: Rosenberg RN, Pascual JM, eds. Rosenberg's Molecular and Genetic Basis of Neurological and Psychiatric Disease. 5th ed. London: Academic Press; 2015 955-961

2 Li Z, Feng T, Teng H, Hu Y, Yao Y, Liu Y. Suprasellar hemangioblastoma without von Hippel-Lindau disease: a case report and literature review. Int J Clin Exp Pathol 2015;8(6):7553-7558

3 Mills SA, Oh MC, Rutkowski MJ, Sughrue ME, Barani IJ, Parsa AT. Supratentorial hemangioblastoma: clinical features, prognosis, and predictive value of location for von Hippel-Lindau disease. Neuro-oncol 2012;14(8):1097-1104

4 O'Reilly GV, Rumbaugh CL, Bowens M, Kido DK, Naheedy MH. Supratentorial haemangioblastoma: the diagnostic roles of computed tomography and angiography. Clin Radiol 1981;32(4):389-392

5 Grisoli F, Gambarelli D, Raybaud C, Guibout M, Leclercq T. Suprasellar hemangioblastoma. Surg Neurol 1984;22(3):257-262

6 Niemelä M, Lim YJ, Söderman M, Jääskeläinen J, Lindquist C. Gamma knife radiosurgery in 11 hemangioblastomas. J Neurosurg 1996;85(4):591-596

7 Kouri J, Chen MY, Watson JC, Oldfield EH. Resection of suprasellar tumors by using a modified transsphenoidal approach. Report of four cases. J Neurosurg 2000;92(6):1028-1035

8 Goto T, Nishi T, Kunitoku N, et al. Suprasellar hemangioblastoma in a patient with von Hippel-Lindau disease confirmed by germline mutation study: case report and review of the literature. Surg Neurol 2001;56(1):22-26

9 Ikeda M, Asada M, Yamashita H, Ishikawa A, Tamaki N. A case of suprasellar hemangioblastoma with thoracic meningioma [in Japanese]. No Shinkei Geka 2001;29(7):679-683

10 Wasenko JJ, Rodziewicz GS. Suprasellar hemangioblastoma in Von Hippel-Lindau disease: a case report. Clin Imaging 2003;27(1):18-22

11 Peker S, Kurtkaya-Yapicier O, Sun I. Sav A, Pamir MN. Suprasellar haemangioblastoma. Report of two cases and review of the literature. J Clin Neurosci 2005;12(1):85-89

12 Fomekong E, Hernalsteen D, Godfraind C, D'Haens J, Raftopoulos C. Pituitary stalk hemangioblastoma: the fourth case report and review of the literature. Clin Neurol Neurosurg 2007;109(3):292-298
13 Miyata S, Mikami T, Minamida Y, Akiyama Y, Houkin K. Suprasellar hemangioblastoma. J Neuroophthalmol 2008;28(4):325-326

14 Lonser RR, Butman JA, Kiringoda R, Song D, Oldfield EH. Pituitary stalk hemangioblastomas in von Hippel-Lindau disease. J Neurosurg 2009;110(2):350-353

15 Peyre M, David P, Van Effenterre R, et al. French NCI Network VHL Disease and Inherited Predisposition to Kidney Cancer. Natural history of supratentorial hemangioblastomas in von Hippel-Lindau disease. Neurosurgery 2010;67(3):577-587, discussion 587

16 Cao Y, Gao P, Wang S, Zhao J. Pituitary infundibulum hemangioblastoma detected by dynamic enhancement MRI. Can J Neurol Sci 2010;37(5):697-699

17 Schär RT, Vajtai I, Sahli R, Seiler RW. Manifestation of a sellar hemangioblastoma due to pituitary apoplexy: a case report. J Med Case Reports 2011;5(496):496

18 Ajler P, Goldschmidt E, Bendersky D, et al. Sellar hemangioblastoma mimicking a macroadenoma. Acta Neurol Taiwan 2012;21(4):176-179

19 Xie T, Zhang X, Hu F, et al. Suprasellar hemangioblastoma mimicking a craniopharyngioma: result of extended endoscopic transsphenoidal approach-case report. Neurol Med Chir (Tokyo 2013;53(10):735-739

20 Lee GI, Kim JM, Choi KS, Kim CH. Sporadic hemangioblastoma in the pituitary stalk: a case report and review of the literature. J Korean Neurosurg Soc 2015;57(6):465-468

21 Kosty J, Staarman B, Zimmer LA, Zuccarello M. Infundibular hemangioblastoma in a patient with neurofibromatosis type 1: case report and review of the literature. World Neurosurg 2016;88:693.e7-693.e12

22 Wenting J, Ogawa Y, Ito J, Tominaga T. Suprasellar hemangioblastoma unrelated to von Hippel-Lindau disease successfully treated through extended transsphenoidal approach: diagnostic value of Von Hippel-Lindau disease gene-derived protein. J Neurol Surg A Cent Eur Neurosurg 2017;78(3):296-301

23 Sizdahkhani S, Feldman MJ, Piazza MG, et al. Somatostatin receptor expression on von Hippel-Lindau-associated hemangioblastomas offers novel therapeutic target. Sci Rep 2017;7:40822

24 Pakdaman MN, Austin MJ, Bannykh S, Pressman BD. Sporadic hemangioblastoma arising from the infundibulum. J Radiol Case Rep 2017;11(5):1-6

25 Alshafai N, Maduri R, Shail M, Chirchiglia D, Meyronet D, Signorelli F. Surgical approach for suprasellar hemangioblastomas preserving the pituitary stalk: review of the literature and report of a further case. Clin Neurol Neurosurg 2018;168:147-152

26 Hattori Y, Tahara S, Yamada O, Yamaguchi M, Ishisaka E, Morita A. Suprasellar hemangioblastoma with reversible edema-like change along the optic tract: a case report and literature review. World Neurosurg 2018;114:187-193

27 Zada G, Lopes MBS, Mukundan S Jr, Laws E Jr, Craniopharyngiomas. In: Zada G, Lopes MBS, Mukundan Jr. S, Laws Jr. E, eds. Atlas of Sellar and Parasellar Lesions. Cham: Springer International Publishing Switzerland; 2016 197-210

28 Crotty TB, Scheithauer BW, Young WF Jr, et al. Papillary craniopharyngioma: a clinicopathological study of 48 cases. J Neurosurg 1995;83(2):206-214

29 Zada G, Lopes MBS, Mukundan S Jr, Laws E Jr, Meningioma of the sellar and parasellar region. In: Zada G, Lopes MBS, Mukundan Jr. S, Laws Jr. E, eds. Atlas of Sellar and Parasellar Lesions. Cham: Springer International Publishing Switzerland; 2016 259-270

30 Kunimatsu A, Kunimatsu N, Kamiya K, Katsura M, Mori H, Ohtomo K. Variants of meningiomas: a review of imaging findings and clinical features. Jpn J Radiol 2016;34(7):459-469 
31 Zada G, Lopes MBS, Mukundan S Jr, Laws E Jr Hemangiopericytoma. In: Zada G, Lopes MBS, Mukundan Jr. S, Laws Jr. E, eds. Atlas of Sellar and Parasellar Lesions. Cham: Springer International Publishing Switzerland; 2016 271-274

32 Han MH, Cho YD, Kim YD, Kin DH. Recurrent sellar and suprasellar hemangiopericytoma. J Korean Neurosurg Soc 2007; $41: 425-428$

33 Jalali R, Srinivas C, Nadkarni TD, Rajasekharan P. Suprasellar haemangiopericytoma-challenges in diagnosis and treatment. Acta Neurochir (Wien 2008;150(1):67-71

34 Pomper MG, Passe TJ, Burger PC, Scheithauer BW, Brat DJ. Chordoid glioma: a neoplasm unique to the hypothalamus and anterior third ventricle. AJNR Am J Neuroradiol 2001;22(3):464-469

35 Gupta S, Bi WL, Mukundan S, Al-Mefty O, Dunn IF. Clinical applications of dynamic CT angiography for intracranial lesions. Acta Neurochir (Wien 2018;160(4):675-680

36 Bi WL, Brown PA, Abolfotoh M. Al-Mefty O, Mukundan S Jr, Dunn IF. Utility of dynamic computed tomography angiography in the preoperative evaluation of skull base tumors. J Neurosurg 2015;123(1):1-8
37 Villablanca JP, Jahan R, Hooshi P, et al. Detection and characterization of very small cerebral aneurysms by using 2D and 3D helical CT angiography. AJNR Am J Neuroradiol 2002;23(7):1187-1198

38 Takhtani D, Dundamadappa S, Puri AS, Wakhloo A. Flow artifact in the anterior communicating artery resembling aneurysm on the time of flight MR angiogram. Acta Radiol 2014;55(10):1253-1257

39 Huang RY, Bi WL, Griffith B, et al; International Consortium on Meningiomas. Imaging and diagnostic advances for intracranial meningiomas. Neuro-oncol 2019;21(Suppl 1) :i44-i61

40 Nishimura S, Hirai T, Shigematsu Y, et al. Evaluation of brain and head and neck tumors with 4D contrast-enhanced MR angiography at 3T. AJNR Am J Neuroradiol 2012;33(3):445-448

41 Ene CI, Xu D, Morton RP, et al. Safety and efficacy of preoperative embolization of intracranial hemangioblastomas. Oper Neurosurg (Hagerstown 2016;12(2):135-140

42 Sawin PD, Follett KA, Wen BC. Laws ER Jr. Symptomatic intrasellar hemangioblastoma in a child treated with subtotal resection and adjuvant radiosurgery. Case report. J Neurosurg 1996;84(6):1046-1050 


\section{Appendix A: Materials and Methods}

The computed tomography angiography (CTA) was obtained using a Toshiba Aquilion One 320-slice multidetector computed tomography scanner. As per protocol, $50 \mathrm{~mL}$ of Isovue (iopamidol $300 \mathrm{mg} / \mathrm{mL}$ of iodine; Bracco Diagnostics Inc., Monroe Township, New Jersey, United States) contrast material was infused. A total of 24 sequential volumes covering the entire brain were acquired at $0.5 \mathrm{~mm}$ thickness that covers $16 \mathrm{~cm}$ of the head in a $z$ plane. The protocol includes a series of intermittent volume scans over a period of 60 seconds. The first volume is used as the mask for the dynamic subtraction. A series of low-dose scans are obtained, first for every 2 seconds during the arterial phase, and then are spaced out to every 5 seconds to capture the slower venous flow. The $\mathrm{mA}$ exposure is increased during the peak arterial enhancement to provide superior three-dimensional (3D) images of the intracranial arteries for a maximum of $310 \mathrm{~mA}(\mathrm{kV} 80)$. The raw data are reconstructed into a dynamic volume imaging to provide a true four-dimensional computed tomography angiography (4D CTA) and digital subtraction angiography (DSA) image of the intracranial circulation. 